\title{
Prevention of the second stage of epithelial loss is a potential novel treatment for bronchiolitis obliterans
}

\author{
Yunge Zhao, MD, PhD, John F. Steidle, BA, Gilbert R. Upchurch, MD, Irving L. Kron, MD, and \\ Christine L. Lau, MD
}

Objectives: Loss of epithelial cells is one of the key factors that lead to airway fibrosis. Loss of epithelial cells
may decrease the barrier to host cell infiltration into the lumen, allowing deposition of extracellular matrix, with
subsequent obliteration of the airway. The objective of this study was to determine whether injection of epithelial
cells/progenitor cells from the recipient into the lumen of the donor trachea could prevent bronchiolitis obliter-
ans (BO) in a mouse heterotopic tracheal transplantation (HTT) model.

Methods: A major histocompatibility complex class I and class II mismatch of mouse HTT model of BO was used. Epithelial cells from recipient mice were isolated and reinjected into the lumen of the allografts on day 3 after transplantation. Rag-1 knock-out and isografts were also performed as controls. The grafts were analyzed by immunohistochemistry and densitometric analysis.

Results: The results demonstrated that tracheal epithelium was lost by day 3, regenerated between 3 to 7 days, and was lost again in all allografts, but not in the isografts or in Rag-1 knock-out groups by day 12 . The reconstituted epithelium was donor originated on day 7 based on green fluorescent protein staining. Furthermore, with the injection of recipient cells into the tracheal lumen, loss of the epithelium was not observed and the luminal obliteration was significantly less in the allografts.

Conclusions: Injection of recipient epithelial cells prevents the second phase of epithelial loss and significantly decreases BO development in an HTT model. Clinically, the use of injected recipient epithelial cells could be a novel treatment for BO. (J Thorac Cardiovasc Surg 2013;145:940-7)

Supplemental material is available online.

Lung transplantation is currently recognized as the preferred treatment for selected patients with end-stage pulmonary diseases. Despite notable advances since its inception, long-term mortality of lung recipients is still highest among all solid organs transplanted. The Achilles' heel of lung transplantation remains chronic allograft rejection. ${ }^{1,2}$ Chronic lung allograft rejection is also called bronchiolitis obliterans (BO) and histologically is a temporal process with loss of luminal epithelium, peribronchiolar leukocyte infiltration, and subsequent fibro-obliteration of the

From the Department of Surgery, University of Virginia Health System, Charlottesville, Va.

C.L.L. is supported by a grant sponsored by the National Heart, Lung, and Blood Institute (1K08HL094704). I.L.K. is supported by 5RO1HL092953 from the National Institutes of Health. G.R.U. is supported by 5RO1HL081629-06 from the National Institutes of Health.

Disclosures: Authors have nothing to disclose with regard to commercial support.

Received for publication April 19, 2012; revisions received July 9, 2012; accepted for publication July 31, 2012; available ahead of print Aug 30, 2012.

Address for reprints: Christine L. Lau, MD, Department of Surgery, PO Box 800679 , Charlottesville, VA 22908-0679 (E-mail: cll2y@ virginia.edu). 0022-5223/\$0.00

Published by Elsevier Inc. on behalf of The American Association for Thoracic Surgery

http://dx.doi.org/10.1016/j.jtcvs.2012.07.098 airways. ${ }^{2,3}$ Once BO develops, progressive decline in pulmonary function is typical. Unfortunately, BO responds poorly to standard immunosuppression, and most patients die of respiratory failure within 5 years of onset.

$\mathrm{We}^{4}$ and others ${ }^{6}$ have used a preclinical, widely described mouse heterotopic tracheal transplant (HTT) model to better understand the mechanisms involved in BO. It is known from these studies that once the epithelial cells are lost, the basement membrane is exposed and fibroblasts/ myofibroblasts infiltrate into lumen and secrete extracellular matrix, with the subsequent development of fibrosis. Mukaida and associates ${ }^{7}$ reported that the regenerated epithelial cells were of donor phenotype on day 10 but changed to recipient phenotype after day 50 in a cryopreserved tracheal allotransplantation dog model. Similarly, Ikonen, ${ }^{8}$ Adams, ${ }^{9}$ and their associates demonstrated that regenerated epithelial cells were of donor phenotype on day 7 but switched to recipient phenotype on days 30 and 60 in orthotopic tracheal allografts in a nonimmunosuppressed rat model. The origin of regenerated epithelial cells has not been reported in the mouse tracheal transplantation model. Some have proposed that circulating recipient epithelial cells may also contribute to repair. ${ }^{10,11}$ Published data have shown that stem cells are involved in airway epithelium repair. ${ }^{12-14}$ We hypothesized that a chimeric epithelium (of donor and recipient origin) would be less immunogenic and potentially be protective against the 


\section{Abbreviations and Acronyms \\ $\mathrm{BO}=$ bronchiolitis obliterans \\ $\mathrm{GFP}=$ green fluorescent protein \\ $\mathrm{HTT}=$ heterotopic tracheal transplant \\ $\mathrm{KO}=$ knock-out}

secondary loss of epithelium. The purpose of this study was to test our hypothesis that the prevention of the secondary loss of epithelial cells could prevent BO development. To prevent this secondary loss, we injected recipient epithelial cells into the donor tracheas after the initial loss and during the regenerative phase with the goal of creating a chimeric epithelium that would be more tolerant. In this study, we tried to explore whether injection of epithelial progenitor cells from recipients into the donor trachea would prevent BO development by preserving the second loss of epithelium cell.

\section{MATERIALS AND METHODS}

\section{Animals}

For tracheal transplant experiments, Balb/c male mice, C57BL6 male mice were purchased from Jackson Laboratory, Bar Harbor, Maine. Green fluorescent protein (GFP; C57BL6 background) male mice were from Linden's Laboratory (28-35 g). Rag-1 knock-out (KO, C57BL6 background) male mice were kindly donated from the laboratory of Dr Victor Laubach. According to the published results, Rag-1 KO mice have no functional $\mathrm{T}$ and $\mathrm{B}$ cells. ${ }^{15}$ However, they have normal levels of functional natural killer cells. All the experimental mice received humane care in accordance with the "Principles of Laboratory Animal Care," formulated by the National Society for Medical Research, and "The Guide for the Care and Use of Laboratory Animals," prepared by the National Academy of Science and published by the National Institutes of Health. The study protocol was approved by the Animal Care and Use Committee at the University of Virginia before experimentation.

\section{Mouse Model of HTT}

We used a heterotopic subcutaneous tracheal transplant model of BO as described in our previous publications. ${ }^{4,5}$ In brief, a major histocompatibility complex class I and class II mismatch was produced by tracheal transplanting between Balb/c $\left(\mathrm{H}-2^{\mathrm{d}}\right)$ tracheas and $\mathrm{C} 57 \mathrm{BL} / 6$ or the other way around.

\section{Experimental Group Design}

Experimental mice were divided into 4 groups: (1) Balb/c tracheas transplanted to C57BL/6, GFP, and Rag-1 KO; (2) GFP tracheas transplanted to Balb/c mice; (3) Balb/c tracheas transplanted to GFP mice treated with GFP-epithelial cells; (4) C57BL/6 tracheas transplanted to $\mathrm{C} 57 \mathrm{BL} / 6$ as isograft controls. In all groups, 4 donor tracheas were transplanted into 1 recipient; 3 recipients were used in each group and each time point (days 3, 7, 12, 21, and 28). A total of 60 donors and 15 recipients were used in each group. The mice were humanely killed and the isograft and allografts were collected on days 3, 7, 12, 21, or 28 for histologic examination and immunhistochemical staining.

\section{Isolation of Murine Tracheal Epithelial Cells}

Mice (C57BL/6 or GFP, the same strain of mice as recipients) were humanely killed and a total of 12 tracheas were isolated and washed with
HEPES-DMEM* buffer supplemented with $1 \%$ penicillin-streptomycin (Invitrogen, Grand Island, NY). The isolated trachea was placed into a 60mm cell culture dish. Next, $50 \mu \mathrm{L}$ of digestion media, which contains DMEM with $0.01 \%$ collagenase A (Roche Diagnostics, Mannheim, Germany) and $1 \%$ penicillin-streptomycin, was injected into the lumen of the isolated trachea and incubated for 1 hour in $5 \%$ carbon dioxide at $37^{\circ} \mathrm{C}$. The tracheas were turned over every 15 minutes. Then the lumina were flushed with 1XPBS containing $1 \%$ penicillin-streptomycin. The collected epithelium-rich solution was centrifuged at $600 \mathrm{rpm}$ for 5 minutes. The cells were confirmed by staining with keratins (K14 and K5) antibodies and cell numbers were counted using a hemacytometer under microscopy. Then the cells from each trachea were resuspended in phosphate-buffered saline solution $(30 \mu \mathrm{L})$, which is supplied with $1 \%$ penicillin-streptomycin. The digested tracheas were immediately fixed in $4 \%$ zinc-formalin. After 24 hours, they were embedded in paraffin, sectioned, and stained for histologic examination.

\section{Injection of the Isolated Epithelial Cells into the Lumen of the Allograft}

On the third day after tracheal transplantation, the recipient mice were anesthetized. An incision was made to expose the transplanted trachea followed by the injection of the isolated epithelial cells directly into the lumen of the allografts. The isolated epithelial cells $(30 \mu \mathrm{L})$ from each trachea were injected into 1 allograft only with a syringe. The same volume (30 $\mu \mathrm{L}$ ) of 1XPBS supplied with $1 \%$ penicillin-streptomycin was also injected into each allograft in the control group.

\section{Histology}

Transplanted tracheal tissues were collected and immediately fixed in $4 \%$ zinc-formalin, After 24 hours they were embedded in paraffin, sectioned, and stained with hematoxylin and eosin or with anti-mouse macrophage (Mac-2; Accurate Chemical \& Scientific Corporation, Westbury, NY), anti-neutrophil, anti-CD3, and anti-GFP antibodies. Collagen deposition was detected by direct red 80 collagen staining.

Immunohistochemical staining of macrophages and neutrophils. Macrophages and neutrophils were detected by immunohistochemical analysis as described previously. ${ }^{4,5}$ In brief, rat anti-mouse neutrophil (AbD Serotec, Raleigh, NC) or rat Mac-2 antibodies were used as primary antibodies. Alkaline phosphatase-conjugated anti-rat immunoglobulin G (Sigma Chemical Company, St Louis, Mo) was used as secondary antibody. Fast-Red (Sigma) was used as substrate. Purified normal rat immunoglobulin G (eBioscience Inc, San Diego, Calif) was used as a negative control. The sections were counterstained lightly with hematoxylin for viewing negatively stained cells. Macrophage and neutrophil infiltration was semiquantified using Image-Pro Plus software (Media Cybernetics, Rockville, Md). The densitometric value of positive staining area of each section was blindly selected and measured.

Immunohistochemical staining of $\mathbf{C D 3}+\mathbf{T}$ cells. The staining was performed according to our previous publications. ${ }^{4,5,16}$ In brief, the transplanted tracheal sections $(5 \mu \mathrm{m})$ were dehydrated and incubated with $1 \%$ hydrogen peroxide. Sections were then boiled in Unmasking Solution (Vector Laboratories, Burlingame, Calif) and blocked with 10\% serum. Goat anti-mouse CD3 $\varepsilon$ antibody (Santa Cruz Biotechnology, Santa Cruz, Calif) is a primary antibody. After incubation with an avidin-biotin complex, immunoreactivity was visualized by incubating the sections with 3 , 3-diaminobenzidine tetrahydrochloride (Dako Corporation, Carpinteria, Calif) to produce a brown precipitate and then counterstained with hematoxylin. The number of CD3+T cells per high-power field was assessed by immunohistochemical staining of tracheal sections, and at least 5 fields

*HEPES = 4-(2-hydroxyethyl)-1-piperazineethanesulfonic acid; DMEM = Dulbecco's modified Eagle's medium. 

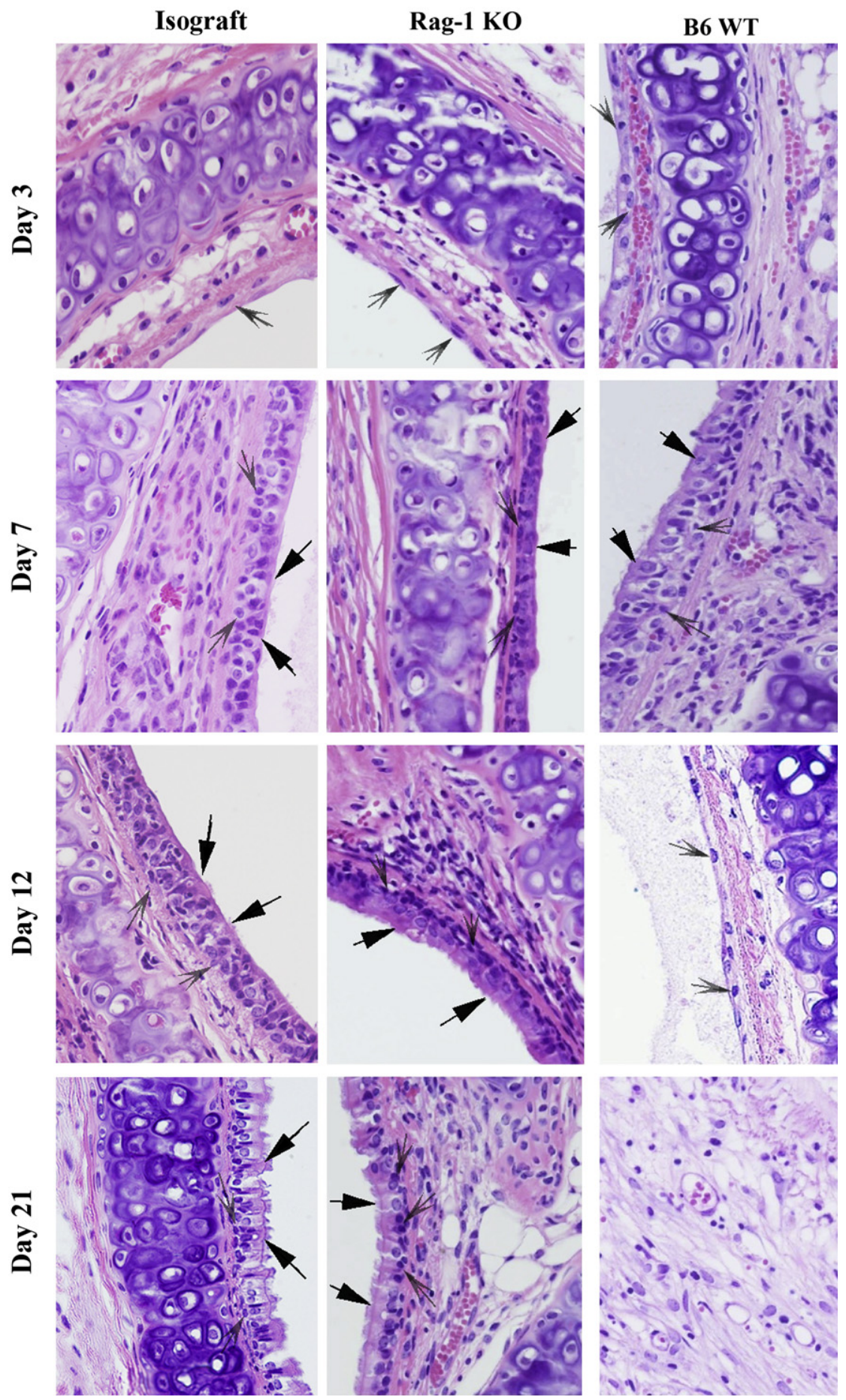

FIGURE 1. Representative pictures of hematoxylin and eosin staining of the allograft from $3,7,12$, and 21 days after transplantation. The magnification of all the pictures was $40 \times$. Black arrows indicate epithelial cells. The gray arrows indicate basal cells. The donors are Balb/c mice and the recipients are Rag-1 $\mathrm{KO}$ and C57BL/6 wild-type mice. C57BL/6 to C57BL/6 isograft controls are also performed.

were counted per trachea by blinded observers. The average cell number was used for statistical analysis.

Measurement of the luminal obliteration. The luminal obliteration was evaluated according to our previous publications. ${ }^{4,5}$ In brief, allografts were photographed at $4 \times$ magnification and the area of the obliterated lumen and the total area of lumen were measured using the Image-Pro Plus software. The percent of the obliteration was calculated by the area of fibrosis divided by the total area of lumen. Eight to 10 allografts were measured in each group. The data were used for statistical analysis. 


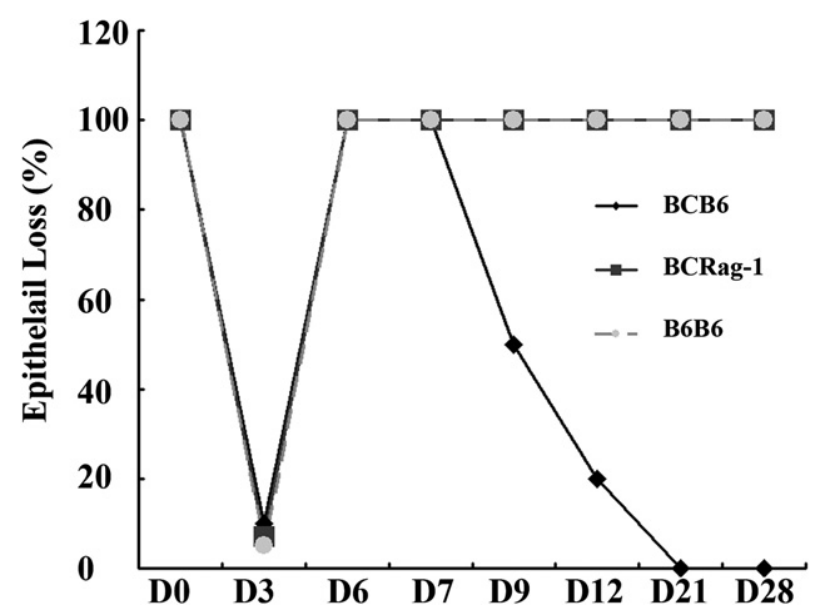

FIGURE 2. Losing of epithelial cells in the transplanted trachea. Epithelial cells are disappearing rapidly owing to ischemia-reperfusion injury in the early stage (days 1-3 after tracheal transplantation) in all transplanted trachea. The pseudoepithelial cells are regenerated from days 4 to 7 in all allografts. The second loss of the regenerated epithelium occurred from days 7 to 14 in all of the allografts except in Rag-1 KO mice, which show a pattern similar to that of isograft controls.

\section{Statistical Analysis}

Data are presented as the mean \pm standard error of the mean. The macrophage, neutrophils, and $\mathrm{CD} 3+\mathrm{T}$ cell counts were compared using 1-way analysis of variance followed by the Student $t$ test for unpaired data with Bonferroni correction. Square roots of tissue cell counts were compared using 1-way analysis of variance.

\section{RESULTS}

\section{Loss of Epithelial Cells in Allografts}

Using a widely established HTT model (Balb/C trachea as donor and wild-type C57BL/6), we have observed the histologic kinetics of allografts after transplantation from the different groups and different time points. The results confirmed our previous finding that the first complete loss of the epithelium occurred in all transplanted tracheas on day 3 after transplantation (Figures 1 and 2). The cause of the first (phase I) epithelial loss is early ischemia-reperfusion injury, and thus this also happened in Rag-1 KO mice and the isograft control group (C57BL/6 into C57BL/6; Figures 1 and 2). There was full reepithelialization of the basement membrane between 3 to 7 days in all of the transplants. Interestingly, the second loss of epithelial cells (phase II) was found in C57BL/6 WT mice from day 7 to day 12 (Figures 1 and 2). In contrast, there was no second loss of epithelial cells in Rag-1 KO mice and isograft controls (Figures 1 and 2). The results show that the second loss of epithelial cells on day 12 is closely correlated with luminal obliteration on day 21 (Figures 1 and 2).

\section{The Regenerated Epithelium Originates From Donor Basal Cells, Not From a Recipient Source}

To determine the origin of the regenerated epithelial cells, we used genetic GFP mice (on C57BL/6 background). First, we confirmed that the cells in trachea, lung, small intestine, spleen, heart, and kidney (data not shown) from GFP mice are GFP-positive cells. Then, using $\mathrm{Balb} / \mathrm{C}$ as donors and GFP mice as recipients, we found that the regenerated epithelial cells in the allografts are GFPnegative (Figure 3, $A$ ), evidence that these cells are of donor origin. We did note a small portion of GFP-positive cells in the epithelial layer, but these were verified to be $\mathrm{CD} 3+\mathrm{T}$ cells (Figure 4, A). Confirming this result, we found when we used GFP mice as donors and Balb/C as recipients, the regenerated epithelial cells were all GFP-positive, evidence again that they are again of donor origin (Figure 3, B). These results indicate that the regenerated epithelial cells originate from the donor, not from the recipients. Of note, in the isografts there were no inflammatory or $\mathrm{T}$ cells seen infiltrating in the epithelial layer in the isograft (Figure 4, B).

\section{Leukocyte Infiltration}

Macrophages infiltration was observed with a peak on day 7 in the allografts of C57BL/6 wild-type mice, whereas the macrophage infiltration reached a peak on day 12 in Rag-1 KO mice (data not shown). However, the neutrophil infiltration peak was on day 7 in all the allografts except on day 3 in Rag-1 KO mice. Interestingly, the neutrophil infiltration vanished after day 7 in Rag-1 KO mice. These findings indicate that the early infiltration (days 1-3) of the macrophage and neutrophil were a reaction of acute injury caused by ischemia-reperfusion. The CD3+ T cells may play an important role in later neutrophil infiltration (days 4-21), because Rag-1 KO mice had no neutrophil infiltration during this time period. The macrophage infiltration was delayed in Rag-1 KO mice.

To determine whether there is an adaptive immune role of $\mathrm{CD} 3+\mathrm{T}$ cell in development of $\mathrm{BO}$, we assessed infiltration of CD3+ T cells via immunohistochemistry in the different groups. The results showed that few $\mathrm{CD} 3+\mathrm{T}$ cells were detected in allografts during the first 3 days after transplantation. Increasing infiltration of $\mathrm{CD} 3+\mathrm{T}$ cells was apparent on day 7 (Figure 4, A), peaked on days 7 to 12 in different allografts, and significantly decreased afterward (data not shown). However, the number of infiltrating CD3+ T cells was significantly abrogated in the epithelial layer of isografts (C57BL/6 to C57BL/6, Figure 4, B). As expected, only few naïve $\mathrm{CD} 3+\mathrm{T}$ cells were detected in Rag-1 KO mice (data not shown). These results suggest that CD3+ T cells infiltrating into the epithelial layer may be a key contributor for second loss of epithelial cells in the transplanted trachea.

\section{Epithelial/basal Cells Harvest for Allograft Injection}

The epithelial cells from recipient mice were isolated using digestion solution with $0.01 \%$ collagenase $\mathrm{A}$ as described in the Methods section. After digestion, the tracheas 

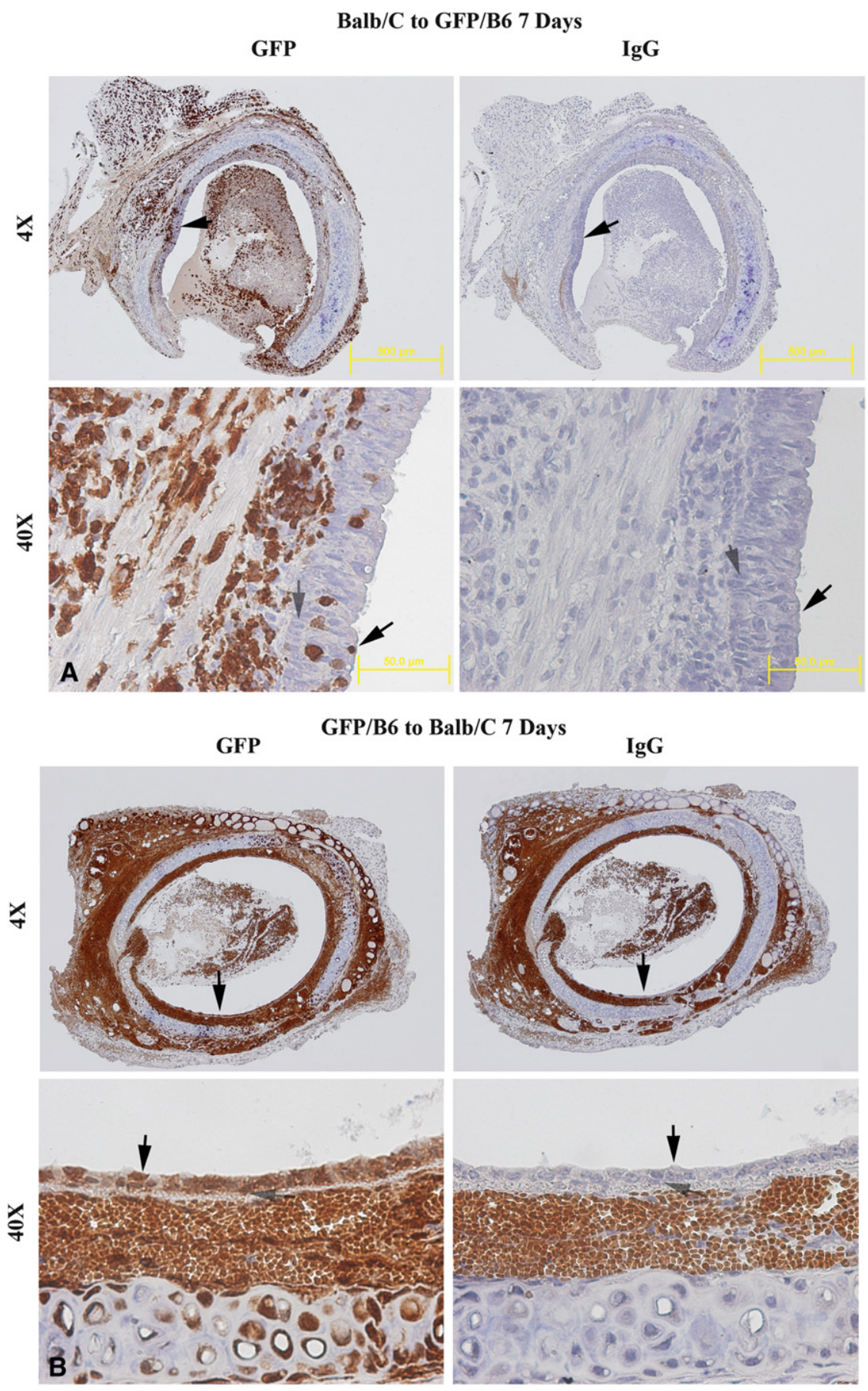

FIGURE 3. Immunohistochemical staining of GFP in allografts on day 7 after tracheal transplantation. A, Representative picture of GFP staining in allograft (Balb/c to GFP/C57BL/6) on day 7. B, Representative picture of GFP staining in allograft (GFP/C57BL/6 to Balb/c) on day 7. Cells stained brown/ dark brown indicate GFP-positive cells. All sections were counterstained lightly with hematoxylin for viewing negatively stained cells. Normal IgG was used as controls. Black arrows indicate epithelial cells. The grey arrows indicate basal cells. The magnifications are indicated in the pictures. GFP, Green fluorescent protein; $\operatorname{Ig} G$, immunoglobulin G.

were processed for histologic examination. As shown in Figure E1, the epithelial cells were not detached within 15 minutes but were detached after 30 minutes. Most of the epithelial cells, including the basal cells, were detached by 1 hour of digestion.
Injection of the Isolated Epithelial Cells From Recipients Prevents the Second Loss of Epithelial Cells and BO Development

After injection of the isolated epithelial cells from recipients into the allografts on day 3 after transplantation, the 

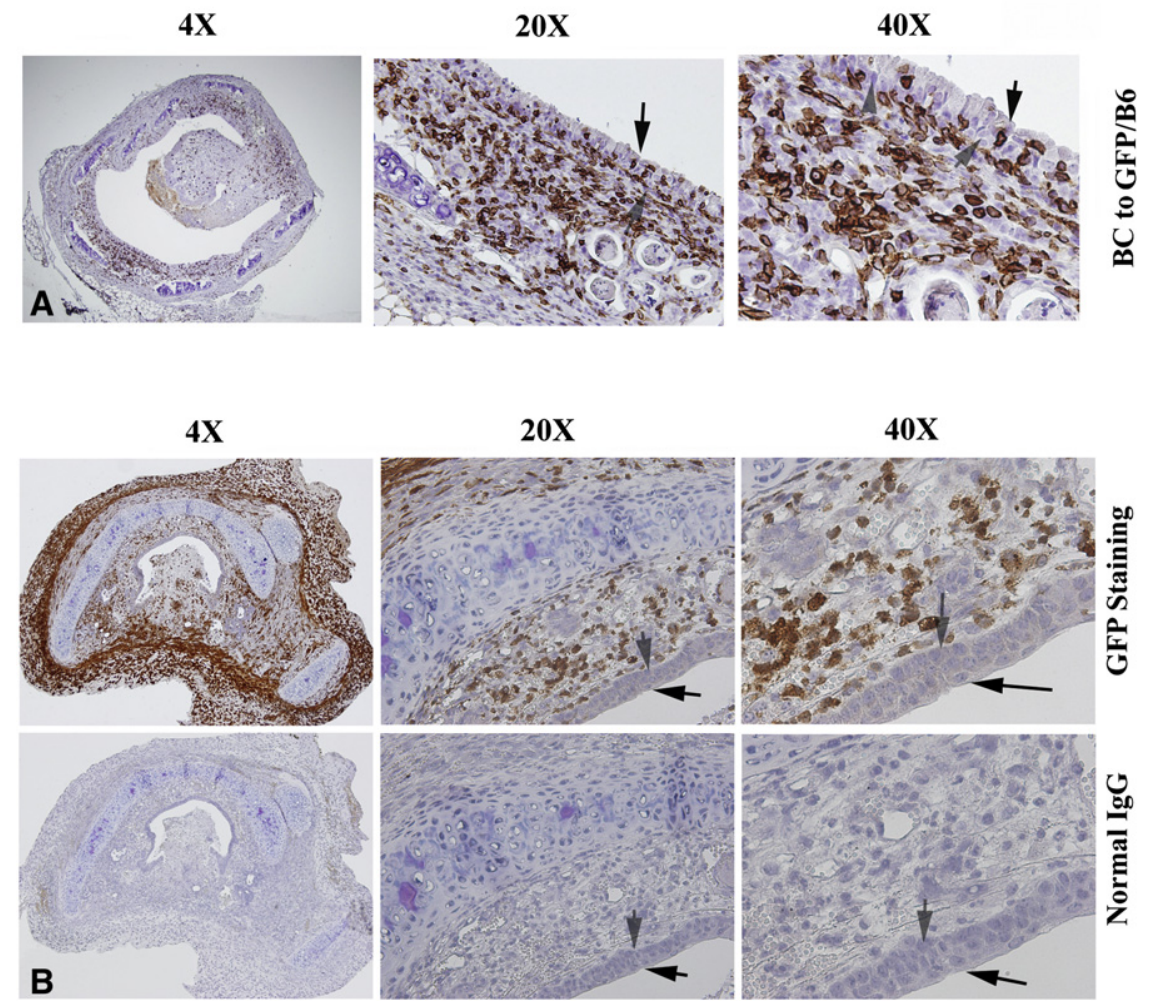

FIGURE 4. Immunohistochemical staining of CD3+T-cells in (A) the allografts of Balb/c to GFP/C57BL/6 and in (B) isograft of C57BL/6 to C57BL/6. Cells stained brown indicate CD3+ T-cell infiltration. All sections were counterstained lightly with hematoxylin for viewing negatively stained cells. The slides were stained with anti-CD3 antibody. Normal IgG was used as control. The magnifications are indicated in the pictures. GFP, Green fluorescent protein; $\operatorname{Ig} G$, immunoglobulin $\mathrm{G} ; B C$, Balb/c.

chimerized pseudoepithelial cells formed. The chimerized epithelial cells did not undergo disappearance when compared with the control groups on day 12 (Figure 5, A). Luminal fibro-obliteration was observed from days 12 to 21 in all the experimental groups except isograft control and Rag-1 KO groups. The luminal obliteration of the allografts that received epithelial injection was significantly less on day $21(P=.007)$ compared with the 1 XPBS control group (Figure $5, B$ ). It is notable that the luminal obliteration was markedly decreased not only in isografts and Rag-1 KO mice, but also in the recipient epithelial cell-injected allografts.

\section{DISCUSSION}

A mouse HTT model used in these studies is often referred to as obliterative airway disease $\mathrm{e}^{3,6}$ because it displays several features that are similar to human BO. Heterotopic tracheas transplanted into HLA-mismatched recipients develop injury that can be divided into an acute phase (days 1-3) characterized by loss of epithelial cells and inflammation resulting from ischemia-reperfusion injury and a later phase (days 4-12) involving the adaptive immune system with lymphocyte infiltration and eventually fibro-obliteration (days 13-28). We acknowledge that the mouse HTT model has significant shortcomings: Most notably, this model is neither vascularized nor aerated and is a large airway model for a small airway disease. There are other models used to study BO, including an orthotopic tracheal model. ${ }^{17}$ However, the orthotopic model is similarly a large airway model, nonvascularized, technically more challenging, and unable to uniformly develop histopathologic BO. A single lung transplantation has been successfully performed in mice ${ }^{18,19}$; however, these transplanted lung allografts have not been shown to reliably develop histopathologic lesions of $\mathrm{BO}$. On the other had, human small airways have an epithelial layer, whereas the mouse small airways lack an epithelial layer; therefore, mouse large airway with the epithelial cells may better represent human small airway in this animal model.

Previous publications have shown the regenerated epithelial cells to be of donor origin in the early stage, but these studies were in rat and dog tracheal transplantation models. ${ }^{7-9}$ Our current study confirmed these results using a mouse HTT model. In this model, there is early loss of epithelial cells (phase I) caused by ischemia-reperfusion injury, followed by regeneration and then a second loss of epithelial cells (phase II), which occurs only in allografts and is believed to be immunologic in origin. Our current result revealed that Rag-1 $\mathrm{KO}$ mice, which lack $\mathrm{T}$ and $\mathrm{B}$ 

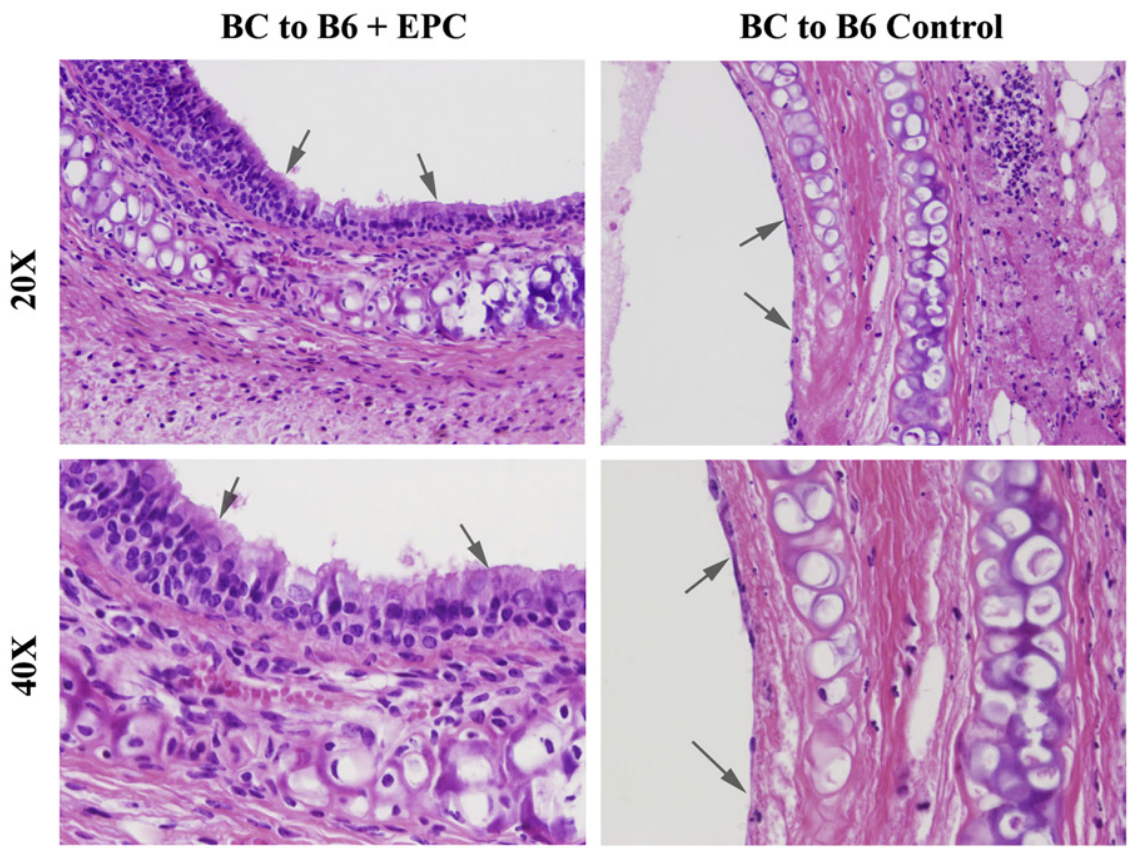

A

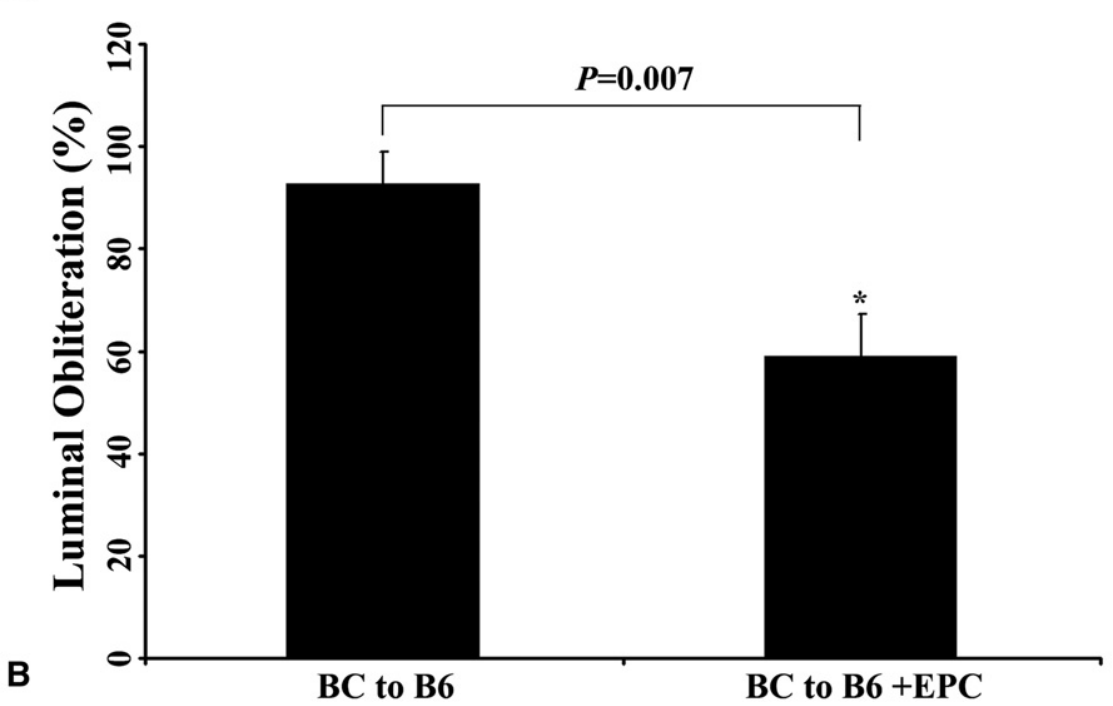

FIGURE 5. A, Injection of recipient epithelial cells prevents the second epithelial loss on day 12 (top panel) when compared with noninjected mice (bottom panel). B, Comparison of luminal obliteration of the allografts with and without epithelial injection on day 21. Data shown are the mean \pm standard deviation. $* P=.007, \mathrm{n}=6 . B C, \mathrm{Balb} / \mathrm{c} ; E P C$, epithelial cells.

lymphocytes, underwent the initial loss of epithelium but not the secondary immunologic loss. Additionally, we used GFP recipients and donors to show the origin of the regenerated epithelial cells to be of donor origin. We also used this strain to detect the integration of injected recipient epithelial cells into the donor trachea. The novel finding of this study is that injection of epithelial cells, which were isolated from recipient trachea, could block BO development by preventing the second loss of epithelial cells in the tested allografts.

Regeneration and restoration of the airway epithelium after physical, viral, or bacterial injury plays a role in numerous respiratory diseases, such as chronic bronchitis, asthma, and cystic fibrosis. ${ }^{20}$ Data from published animal models show epithelial regeneration to be a complex process involving migration of the basal cells adjacent to the injury, rapid restoration of tight junctions followed by squamous metaplasia, active mitosis leading to basal and mucous cell hyperplasia, and progressive redifferentiation with the emergence of ciliated and mucous cells, and ciliogenesis. This process allows for the regeneration of a functional mucociliary epithelium. ${ }^{20}$

Evidence suggests that stem/progenitor cells play an important role in epithelial regeneration after trachea/lung 
injuries. ${ }^{13,21-24}$ The idea and technique of implanting recipient epithelium into a heterotopic donor trachea before orthotopic transplantation was previously published as a successful case report by the Leuven group. ${ }^{25}$ Because the second loss of epithelial cells is associated with alloimmune reaction, we explored the possibility that injection of epithelial stem/progenitor cells from recipients may prevent BO development in the mouse HTT model.

Several potential sources of stem/progenitor cells for airway epithelium have been identified, including endogenous stem/progenitor cells present in the respiratory tract and/or from other tissues in the body; these latter cells can be transported to the lung, where they can divide and grow. ${ }^{13}$ In this study, we focus on the potential function of local epithelial stem/progenitor cells (basal and Clara cells) after tracheal transplantation. Inasmuch as both basal and secretory epithelial cells may serve as stem/progenitors during trachea/lung injuries, we investigated the use of mixed epithelial cells from recipients in our current study. The results demonstrated that injection of tracheal epithelial cells was able to prevent the second loss of epithelial cells and, as a consequence, prevented BO development. Our results provide preclinical evidence that epithelial stem/progenitor cells may be a viable treatment strategy for BO after lung transplantation.

In conclusion, our results show that injection of epithelial stem/progenitor cells obtained from recipients into the allografts on day 3 prevents second loss of epithelial cell in the allografts, which subsequently decreased BO development in a mouse HTT model. These results suggest that using epithelial stem/progenitor cells may provide a novel therapeutic strategy to prevent $\mathrm{BO}$ after lung transplantation.

We thank Dr Joel Linden who provided us with the GFP/B6 mice and Dr Victor Laubach provided us with the Rag-1 KO mice.

\section{References}

1. Granton J. Update of early respiratory failure in the lung transplant recipient. Curr Opin Crit Care. 2006;12:19-24.

2. Chan A, Allen R. Bronchiolitis obliterans: an update. Curr Opin Pulm Med. 2004;10:133-41.

3. McDyer JF. Human and murine obliterative bronchiolitis in transplant. Proc Am Thorac Soc. 2007;4:37-43.

4. Zhao Y, LaPar DJ, Steidle J, Emaminia A, Kron IL, Ailawadi G, et al. Adenosine signaling via the adenosine $2 \mathrm{~B}$ receptor is involved in bronchiolitis obliterans development. J Heart Lung Transplant. 2010;29:1405-14.
5. Lau CL, Zhao Y, Kron IL, Stoler MH, Laubach VE, Ailawadi G, et al. The role of adenosine A2A receptor signaling in bronchiolitis obliterans. Ann Thorac Surg. 2009;88:1071-8.

6. Hele DJ, Yacoub MH, Belvisi MG. The heterotopic tracheal allograft as an animal model of obliterative bronchiolitis. Respir Res. 2001;2:169-83.

7. Mukaida T, Shimizu N, Aoe M, Andou A, Date H, Moriyama S. Origin of regenerated epithelium in cryopreserved tracheal allotransplantation. Ann Thorac Surg. 1998;66:205-8.

8. Ikonen TS, Brazelton TR, Berry GJ, Shorthouse RS, Morris RE. Epithelial regrowth is associated with inhibition of obliterative airway disease in orthotopic tracheal allografts in non-immunosuppressed rats. Transplantation. 2000;70: 857-63.

9. Adams BF, Brazelton T, Berry GJ, Morris RE. The role of respiratory epithelium in a rat model of obliterative airway disease. Transplantation. 2000;69:661-4.

10. Gomperts BN, Belperio JA, Rao PN, Randell SH, Fishbein MC, Burdick MD, et al. Circulating progenitor epithelial cells traffic via CXCR4/CXCL12 in response to airway injury. J Immunol. 2006;176:1916-27.

11. Kleeberger W, Versmold A, Rothamel T, Glockner S, Bredt M, Haverich A, et al. Increased chimerism of bronchial and alveolar epithelium in human lung allografts undergoing chronic injury. Am J Pathol. 2003;162:1487-94.

12. Rock JR, Onaitis MW, Rawlins EL, Lu Y, Clark CP, Xue Y, et al. Basal cells as stem cells of the mouse trachea and human airway epithelium. Proc Natl Acad Sci U S A. 2009;106:12771-5.

13. Roomans GM. Tissue engineering and the use of stem/progenitor cells for airway epithelium repair. Eur Cell Mater. 2010;19:284-99.

14. Giangreco A, Arwert EN, Rosewell IR, Snyder J, Watt FM, Stripp BR. Stem cells are dispensable for lung homeostasis but restore airways after injury. Proc Natl Acad Sci U S A. 2009;106:9286-91.

15. Mombaerts P, Iacomino J, Johnson RS, Herrup K, Tonegawa S, Papaioannou VE. RAG-1-deficient mice have no mature B and T lymphocytes. Cell. 1992;68: 869-77.

16. Zhao Y, Sharma AK, LaPar DJ, Kron IL, Ailawadi G, Liu Y, et al. Depletion of tissue plasminogen activator attenuates lung ischemia-reperfusion injury via inhibition of neutrophil extravasation. Am J Physiol Lung Cell Mol Physiol. 2011; 300:L718-29.

17. Genden EM, Boros P, Liu J, Bromberg JS, Mayer L. Orthotopic tracheal transplantation in the murine model. Transplantation. 2002;73:1420-5.

18. Okazaki M, Krupnick AS, Kornfeld CG, Lai JM, Ritter JH, Richardson SB, et al A mouse model of orthotopic vascularized aerated lung transplantation. Am J Transplant. 2007;7:1672-9.

19. Jungraithmayr WM, Korom S, Hillinger S, Weder W. A mouse model of orthotopic, single-lung transplantation. J Thorac Cardiovasc Surg. 2009;137:486-91.

20. Puchelle E, Peault B. Human airway xenograft models of epithelial cell regeneration. Respir Res. 2000;1:125-8.

21. Inayama Y, Hook GER, Brody AR, Jetten A, Gilmore L, Gray T, et al. In vitro and in vivo growth and differentiation of clones of tracheal basal cells. Am J Pathol. 1989;134:539-49.

22. Ford J, Terzaghi-Howe M. Basal cells are the progenitors of primary tracheal epithelial cell cultures. Exp Cell Res. 1992;198:69-77.

23. Rawlins EL. Lung epithelial progenitor cells: lessons from development. Proc Am Thorac Soc. 2008;5:675-81.

24. Rawlins EL, Okubo T, Que J, Xue Y, Clark C, Luo X, et al. Epithelial stem/progenitor cells in lung postnatal growth, maintenance, and repair. Cold Spring Harb Symp Quant Biol. 2008;73:291-5.

25. Delaere P, Vranckx J, Verleden G, De Leyn P, Van Raemdonck D. Tracheal allotransplantation after withdrawal of immunosuppressive therapy. $N$ Engl J Med. 2010;362:138-45. 

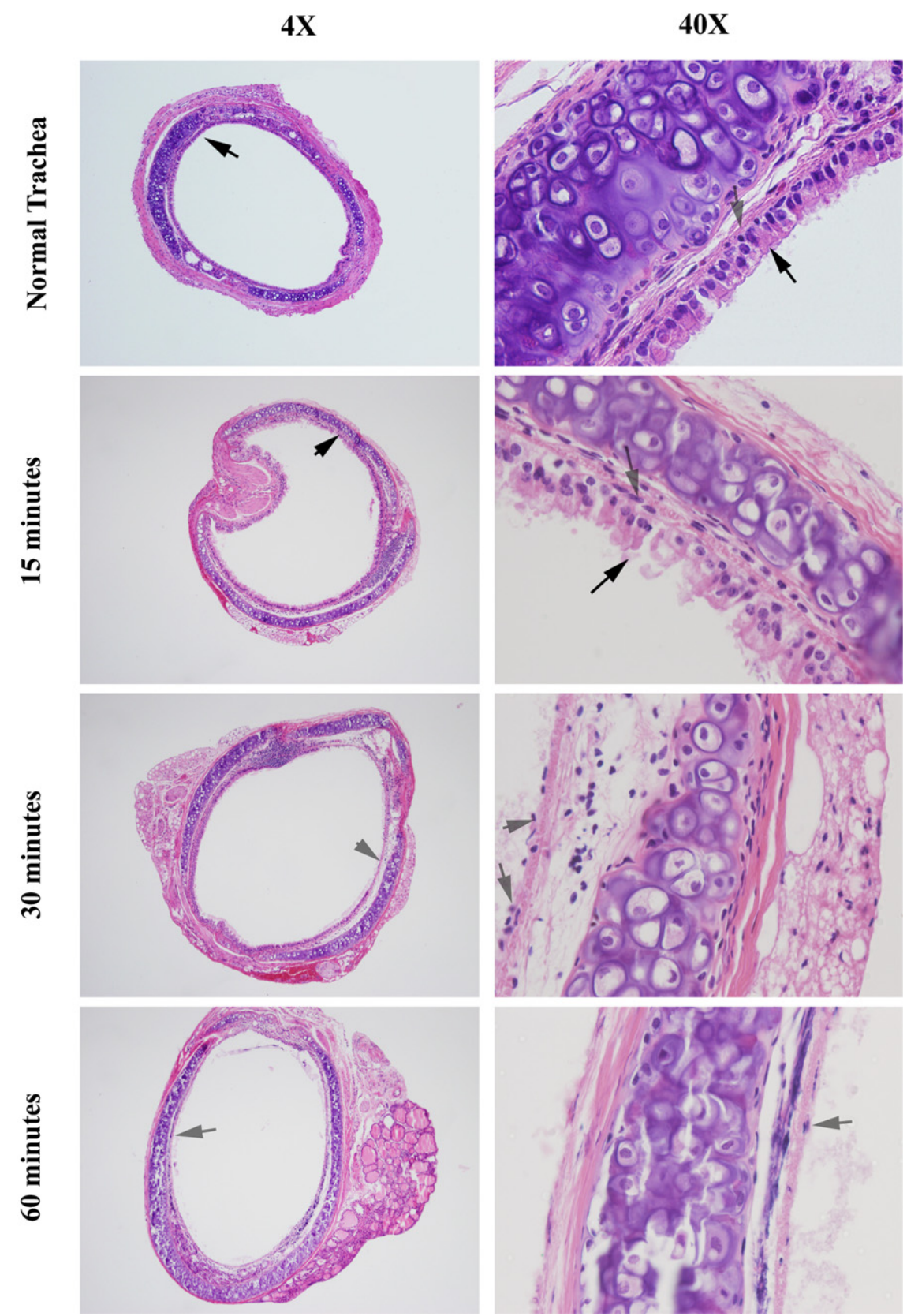

FIGURE E1. Histologic examination of epithelial cells after digestion with $0.01 \%$ collagenase A in the trachea of GFP/C57BL/6 mice. The epithelial layers are intact in the nondigested normal trachea and 15-minutes digestion trachea. Epithelial cells detached after digestion of 30 minutes and completely detached after 1 hour of digestion. Black arrows indicate epithelial cells. The gray arrows indicate basal cells. The magnifications are indicated on the pictures. GFP, Green fluorescent protein. 